\title{
Caracterización de pacientes con demencia de tipo Alzheimer precoz vs tardío
}

\author{
Characterization of patients with early vs. late- \\ onset Alzheimer's dementia
}

\author{
Nohemi Meza-Cely, Vanessa Sabella-Jiménez, Jorge Acosta-Reyes, Carlos Otero-Herrera, \\ María Susana Pérez-Olivo, Dania Ruiz-Plaza, Dennys Jiménez-Hernández, \\ Carlos Silvera-Redondo, Gloria Rolón-Martínez • Barranquilla (Colombia)
}

DOI: https://doi.org/10.36104/amc.2020.1316

\section{Resumen}

Introducción: la demencia de tipo Alzheimer (DTA) tiene presentación precoz o tardía. Es necesaria mayor información sobre factores de riesgo según edad de aparición de DTA. El objetivo es caracterizar variables sociodemográficas, antropométricas, de laboratorio, genéticas y antecedentes en pacientes con diagnóstico de novo de DTA según edad de aparición en el Hospital Universitario C.A.R.I. sede salud mental en un periodo de dos años.

Metodología: estudio descriptivo transversal con 39 pacientes con diagnóstico de novo de DTA. Se realizó un cuestionario, solicitaron paraclínicos y se obtuvo una muestra sanguínea para genotipificación de APOE. Se utilizó el software IBM SPSS 21 para análisis.

Resultados: el $82.05 \%$ tenían DTA tardío y $17.95 \%$ DTA precoz. El $57.14 \%$ con DTA precoz y $71.90 \%$ con DTA tardía eran de sexo femenino. El 71.44\% con DTA precoz eran casados y $53.12 \%$ con DTA tardío eran viudos. Solo $14.29 \%$ con DTA precoz y $18.75 \%$ con DTA tardío tenían niveles óptimos de LDL. El 79.49\% de la población era heterocigoto para el alelo 84 . El $71.43 \%$ con DTA precoz tenía antecedente familiar de demencia.

Discusión: la edad es el principal factor asociado a DTA y el sexo femenino fue más frecuente en ambos grupos. Las relaciones sociales juegan un rol en la identificación temprana de la sintomatología. Las alteraciones del perfil lipídico se evidenciaron en ambos grupos. Tener al menos un alelo $\varepsilon 4$ es un hallazgo frecuente de DTA. Tener un familiar con demencia y/o Alzheimer de primer grado de consanguinidad fue más frecuente en DTA precoz. (Acta Med Colomb 2020; 45. DOI: https://doi.org/10.36104/amc.2020.1316).

Palabras clave: $A P O E$, demencia; enfermedad de Alzheimer, comienzo precoz, enfermedad de Alzheimer, comienzo tardío.

\footnotetext{
Abstract

Introduction: Alzheimer's dementia (AD) has an early and a late onset. More information is needed regarding risk factors according to the age of onset of AD. The objective is to characterize the sociodemographic, anthropometric, laboratory and genetic variables as well as the history of patients with a de novo diagnosis of $\mathrm{AD}$, by age of onset, at the Hospital Universitario C.A.R.I.'s mental health site over a period of two years.

Methods: a cross-sectional descriptive study of 39 patients with a de novo diagnosis of AD. A questionnaire was completed, paraclinical studies were ordered and a blood sample was obtained for APOE genotyping. The IBM SPSS 21 software was used for analysis.

Results: $82.05 \%$ had late-onset and $17.95 \%$ had early-onset AD. Of those with early-onset AD, $57.14 \%$ were females, as were $71.90 \%$ of those with late-onset AD. $71.44 \%$ of those with early-onset $\mathrm{AD}$ were married and $53.12 \%$ with late-onset AD were widowed. Only $14.29 \%$ with early-onset and $18.75 \%$ with late-onset AD had optimal LDL levels. Altogether, $79.49 \%$ of the population was heterozygous for the $\varepsilon 4$ allele. $71.43 \%$ of those with early-onset AD had a family history of dementia.
}

Dr. Nohemí Meza-Cely: Médica, Especialista en Neurología Clínica; Dra.Vanessa SabellaJiménez: Médica; Dr. Jorge Acosta-Reyes: Magister en Ciencias Clínicas, Departamento de Salud Pública; Dr. Carlos Otero-Herrera: Médico; Dra. María Susana Pérez-Olivo: Médica; Dra. Dania Ruiz-Plaza: Residente de Psiquiatría; Dra. Dennys Jiménez-Hernández: Residente de Medicina Interna; Dr. Carlos Silvera-Redondo: Especialista en Genética Médica, Doctorado en Inmunología Médica; Gloria Rolón-Martínez: Técnico de Laboratorio, Maestría en Ciencias Básicas Biomédicas. Departamento de Medicina, Universidad del Norte. Barranquilla (Colombia).

Correspondencia: Dra. Nohemí Meza Cely. Barranquilla (Colombia).

E-mail: nohemimeza1805@hotmail.com Recibido: 26/II/2019 Aceptado: 11/II/2020 
Discussion: age is the main factor associated with $\mathrm{AD}$ and females were more frequent in both groups. Social relationships play a role in early detection of symptoms. Lipid profile abnormalities were seen in both groups. Having at least one $\varepsilon 4$ allele is a frequent finding in AD. Having a firstdegree relative with dementia and/or Alzheimer's was more frequent in early-onset AD. (Acta Med Colomb 2020; 45. DOI: https://doi.org/10.36104/amc.2020.1316).

Key Words: APOE, dementia; Alzheimer's disease, early onset; Alzheimer's disease, late onset.

\section{Introducción}

La enfermedad de Alzheimer (EA) es una enfermedad degenerativa, caracterizada por múltiples déficits cognitivos y deterioro de la capacidad intelectual global, interfiriendo con el funcionamiento ocupacional y social del individuo (1). Se distinguen tres etapas en la evolución de la enfermedad; sin embargo, en principio se reconocieron dos modalidades de EA: una senil y otra presenil, dependiendo de si los síntomas inician antes o después de los 65 años (2). Antes de los 65 años de edad, un $10 \%$ de los pacientes presenta la forma familiar o de inicio precoz, que se ha asociado con patrón de herencia autosómico dominante y mutaciones en diversos genes. Después de los 65 años de edad, la demencia tipo Alzheimer (DTA) se presenta de forma esporádica o tardía, influenciada por factores de susceptibilidad que interactúan con factores medioambientales (1).

La EA es un problema de salud pública mundial, debido a que se estima que más de 50 millones de personas viven con demencia (3). En Colombia la demencia tiene una prevalencia de 1.8-3.4\% en mayores de 65-75 años respectivamente (4). Se evaluaron 2018 pacientes en una clínica en Bogotá, de los cuales 49.56\% se diagnosticaron por consenso como EA (4). Se requieren estrategias y mayor número de estudios nacionales, primordialmente en la región Caribe enfocados en obtener un mayor conocimiento sobre los factores de riesgo, que permitan lograr un abordaje terapéutico adecuado y temprano para mejorar la calidad de vida del paciente (5-7). El objetivo de esta investigación es caracterizar variables sociodemográficas, antropométricas, de laboratorio, genéticas y antecedentes en pacientes con DTA con diagnóstico de novo y según edad de aparición en el Hospital Universitario C.A.R.I. sede salud mental en un periodo de dos años.

\section{Material y métodos}

Se realizó un estudio descriptivo transversal con un muestreo no aleatorio por conveniencia de tipo secuencial de los pacientes diagnosticados con DTA de novo en el Hospital Universitario C.A.R.I. sede salud mental de Barranquilla, Colombia entre 2014-2016. El Comité de Ética de la Universidad del Norte dio el aval para la ejecución de la investigación.

Inicialmente, se diagnosticó EA probable según la sintomatología y clínica del paciente, bajo los criterios del National Institute on Aging, Alzheimer's Association (8) y CIE-10 (9). Entre 10 y 15 días después de la consulta inicial, se realizó el diagnóstico posible de EA según criterios de Alzheimer's Association (8), con base en la valoración neuropsicológica, resultados de imagenología y paraclínicos. No se realizó diagnóstico de EA seguro, debido a que en el transcurso del estudio no se realizaron biopsias postmortem. Se clasificó a los pacientes menores de 65 años como DTA precoz y a los mayores de 65 años como DTA tardío (10). Los criterios de inclusión del estudio fueron: edad mayor a 45 años, tener diagnóstico de novo de DTA por evaluación neuropsicológica y criterios diagnósticos del National Institute on Aging, Alzheimer's Association y CIE-10, ser paciente de consulta externa de Neurología del Hospital Universitario C.A.R.I sede salud mental, vivir en el departamento del Atlántico desde el nacimiento, firmar consentimiento informado y tener acompañante mayor de edad. Se excluyeron pacientes con anomalías estructurales cerebrales u otras entidades causantes de los síntomas del paciente, como demencias mixtas, vasculares, frontotemporales, tumores, deficiencia de vitamina B12, entre otros.

Las variables incluidas fueron características sociodemográficas (edad, sexo, estado civil), medidas antropométricas (I.M.C), medidas de laboratorio (glicemia en ayunas, colesterol total, LDL, HDL y triglicéridos), medidas genéticas (APOE), y antecedentes personales, familiares, patológicos y tóxicos (antecedente de tabaquismo, hipertensión arterial, diabetes mellitus, hiperlipidemia, familiar con demencia y familiar con Alzheimer) en pacientes con diagnóstico de DTA de novo. La información de antecedentes se obtuvo por medio de datos suministrados por autorreporte en un cuestionario estructurado, las medidas de laboratorio, por medio de resultados obtenidos en la entidad promotora de salud según la afiliación de cada paciente, y la genotipificación del gen $A P O E$, a través del análisis de la muestra de sangre extraída por los investigadores. Para obtener el índice de masa corporal (IMC) se implementó una pesa y tallímetro calibrados. Los puntos de corte de para colesterol total, LDL, HDL y triglicéridos fueron categorizados según la guía Adult Treatment Panel III (11). La categoría intermedia de colesterol HDL corresponde a niveles entre 41 $\mathrm{mg} / \mathrm{dL}$ hasta $59 \mathrm{mg} / \mathrm{dL}$. Los puntos de corte para glicemia en ayunas fueron categorizados según la guía Standards of Medical Care in Diabetes (12).

\section{Genotipificación del gen APOE}

Las muestras fueron procesadas y analizadas en el Laboratorio de Genética de la Universidad del Norte. De cada 
paciente se obtuvo una muestra de $5 \mathrm{~mL}$ de sangre periférica en tubo vacutainer con EDTA. El ADN genómico se obtuvo mediante el kit comercial UltraClean TM Blood DNA Isolation $\mathrm{Kit}^{\circledR}$ (Mo Bio Laboratories, Inc.). Para la determinación de los diferentes alelos se amplificó una región del exón 4 del gen APOE que contiene los sitios polimórficos 112 y 158 y es donde ocurren las sustituciones de nucleótidos que dan origen a las diferentes isoformas del $A P O E$, mediante los iniciadores específicos F4 5' -- ACAGAATTCGCCCCGGCCTGGTACAC-3 ' hacia adelante y F6 5'.-TAAGCTTGGCACGGCTGTCCA AGGA-3 ' hacia atrás (Invitrogen Life Technologies, USA), descritos por Hixson y Vernier (13), con lo que se obtuvo un producto amplificado de $244 \mathrm{pb}$.

La amplificación se hizo en el equipo $\operatorname{ICycler}^{\circledR}$ (BioRad), bajo las siguientes condiciones: una desnaturalización inicial a $95^{\circ} \mathrm{C}$ durante tres minutos, seguida de 30 ciclos con una desnaturalización a $95^{\circ} \mathrm{C}$ durante 45 segundos, una hibridación a $62^{\circ} \mathrm{C}$ durante 30 segundos, una extensión a $72^{\circ} \mathrm{C}$ durante 30 segundos y una elongación final a $72{ }^{\circ} \mathrm{C}$ durante siete minutos. Luego se procedió a la digestión de las muestras con cinco unidades de la endonucleasa de restricción HhaI (Gibco-BRL, Rockville, MD, USA) durante 16 horas a $37^{\circ} \mathrm{C}$. Los fragmentos obtenidos se separaron por electroforesis en gel de poliacrilamida al $8 \%$ durante cuatro horas a 60 voltios, y se observaron los diferentes patrones de bandas característicos de cada genotipo. Finalmente se realizó PCR en tiempo real por discriminación alélica TaqMan (Applied Biosystems), utilizándose para la posición 112 el ensayo ID C_3084793_20(rs429358) y para la posición 158 el ensayo ID C_904973_10 (rs7412) en el equipo 7500 Real Time PCR System. Los resultados fueron observados como curvas de amplificación reconocidas a partir del marcaje para cada sonda (VIC/FAM) llevado a cabo en el software 7500 v2.0.1.

\section{Análisis estadístico}

Se realizó un análisis univariado con estadísticos descriptivos, el cual expresó las frecuencias absolutas y relativas de las variables cualitativas, y con medidas de tendencia central y dispersión para variables cuantitativas. La determinación de las frecuencias alélicas y genotípicas se obtuvo mediante conteo directo.

Se categorizaron los pacientes en dos grupos según la edad de presentación, el primero con DTA precoz (menor de 65 años) y el segundo con DTA tardío (mayor o igual de 65 años), para posteriormente determinar la presencia de las variables sociodemográficas, antropométricas, de laboratorio, genéticas y antecedentes. Se utilizó el software IBM SPSS Statistics versión 21.

\section{Control de sesgos}

El cuestionario fue diseñado con vocabulario sencillo y entendible. La información fue suministrada por el paciente y/o el acompañante. De cada muestra de laboratorio se evaluó el valor reportado, teniendo en cuenta el rango de normalidad establecido por cada laboratorio para cada examen. En un paciente se encontró un infarto lacunar no significativo en su imagenología cerebral de control, sin embargo, no se excluyó del estudio, puesto que el diagnóstico de DTA fue realizado bajo valoración neuropsicológica previa con imagenología sin alteración vascular inicial.

\section{Resultados}

La población correspondió a 39 pacientes. El 82.05\% de los pacientes tenían una forma de presentación de DTA tardía, mientras que $17.95 \%$ correspondían a presentación precoz. El promedio de edad de presentación para el grupo de DTA precoz fue de $60.85 \pm 3.48$ años (mediana 62, RIC 57-64). En el grupo tardío fue de $78.87 \pm 5.39$ años (mediana de 78, RIC 76-83). El sexo femenino se evidenció en 57.14$71.90 \%$ de la población con DTA precoz y tardía, respectivamente. Un $71.44 \%$ con DTA precoz eran casados, mientras que $53.12 \%$ con DTA tardía eran viudos. Un $57.14 \%$ con DTA precoz tenían sobrepeso, mientras que $40.63 \%$ con DTA tardío tenía sobrepeso (Tabla 1).

Se observó alteración de glucosa e hiperglicemia en 9.37$6.25 \%$ de los pacientes con aparición tardía. En cuanto al perfil lipídico $42.86 \%$ con aparición temprana y $25 \%$ con presentación tardía presentaron un nivel límite alto de colesterol total, mientras que sólo $18.75 \%$ con DTA tardía tenían niveles altos. En referencia al colesterol HDL, el $42.86 \%$ del grupo precoz tenía nivel intermedio y $28.57 \%$ nivel bajo. El $62.5 \%$ del grupo tardío presentó niveles intermedios y $12.5 \%$ niveles bajos de colesterol HDL. Sólo $14.29 \%$ de DTA precoz obtuvo niveles óptimos de LDL, similar al grupo tardío con $18.75 \%$. Dentro de los triglicéridos $14.29 \%$

Tabla 1. Caracterización de variables sociodemográficas y antropométricas en pacientes con diagnóstico de novo de DTA según edad de aparición en el Hospital Universitario C.A.R.I. sede salud mental en un periodo de dos años.

\begin{tabular}{|l|c|c|c|c|}
\hline \multirow{2}{*}{ Variable } & \multicolumn{4}{|c|}{ Tipo de aparición de EA } \\
\hline \multirow{2}{*}{ Sexo } & \multicolumn{2}{|c|}{ Precoz (n=7) } & \multicolumn{2}{c|}{ Tardío (n=32) } \\
\cline { 2 - 5 } Femenino & Número & $(\%)$ & Número & $(\%)$ \\
\cline { 2 - 5 } Masculino & 4 & 57.14 & 23 & 71.90 \\
\hline Estado civil & 3 & 42.86 & 9 & 28.10 \\
Soltero & 1 & 14.28 & 2 & 6.25 \\
Casado & 5 & 71.44 & 11 & 34.37 \\
Unión libre & 0 & 0.00 & 1 & 3.13 \\
Divorciado & 0 & 0.00 & 1 & 3.13 \\
Viudo & 1 & 14.28 & 17 & 53.12 \\
\hline I.M.C & & & & \\
Bajo peso & 0 & 0.00 & 3 & 9.37 \\
Normal & 3 & 42.86 & 15 & 46.88 \\
Sobrepeso & 4 & 57.14 & 13 & 40.63 \\
Obesidad grado I & 0 & 0.00 & 1 & 3.12 \\
\hline
\end{tabular}


de DTA precoz fueron categorizados tanto límite alto de la normalidad como elevado, mientras que para los individuos con aparición tardía, el $15.63 \%$ fueron límite alto de la normalidad y $9.37 \%$ elevado (Tabla 2 ).

Para el polimorfismo del gen $A P O E$, un $79.49 \%$ de la población general con DTA fueron heterocigotos para el alelo $\varepsilon 4$, de los cuales $64.10 \%$ presentaron $\varepsilon 3 / \varepsilon 4$ y $15.39 \%$ presentaron $\varepsilon 2 / \varepsilon 4$. El $12.82 \%$ de la población con DTA fueron homocigotos para el alelo $\varepsilon 3$ y $2.56 \%$ fueron homocigotos para el alelo $\varepsilon 2$. El $5.13 \%$ eran $\varepsilon 2 / \varepsilon 3$. No se encontraron homocigotos para el alelo $\varepsilon 4$. El $71.44 \%$ de los pacientes con DTA precoz presentaron heterocigosidad alélica para $A P O E \varepsilon 4$, siendo $57.16 \%$ de $\varepsilon 3 / \varepsilon 4$ y $14.28 \%$ de $\varepsilon 2 / \varepsilon 4$. El $81.24 \%$ con DTA tardía presentaron al menos un alelo $A P O E \varepsilon 4$, siendo $65.62 \%$ para $\varepsilon 3 / \varepsilon 4$ y $15.62 \%$ para $\varepsilon 2 /$ $\varepsilon 4$. Se presentó homocigosidad alélica para $A P O E \varepsilon 3$ en un $14.28 \%$ de los precoces, mientras que para el grupo tardío se observó $12.5 \%$ con $\varepsilon 3 / \varepsilon 3$ y $3.13 \%$ con homocigosidad alélica para APOE $\varepsilon 2$ (Tabla 3).

$\mathrm{El}$ antecedente de tabaquismo se evidenció en $57.14 \%$ del grupo precoz y en $59.37 \%$ del tardío. Tanto el $75 \%$ con DTA precoz (ex)tabaquistas, como el $73.68 \%$ de los tardíos (ex)tabaquistas habían fumado por más de cinco años. De la población con DTA precoz, $42.85 \%$ eran hipertensos y $28.57 \%$ tenían hiperlipidemia. De los individuos con DTA tardía, $46.87 \%$ eran hipertensos, $12.5 \%$ eran diabéticos y $25 \%$ tenía hiperlipidemia. El $71.43 \%$ con DTA precoz presentaron antecedente familiar de demencia, observándose el $100 \%$ del antecedente en familiares de primer grado de consanguinidad. Del $59.37 \%$ del grupo tardío con ante- cedente familiar de demencia, el $94.74 \%$ fue de segundo grado de consanguinidad. Igualmente $57.14 \%$ del grupo precoz, presentó familiares con EA, siendo estos de primer grado de consanguinidad. De $43.75 \%$ del grupo tardío con antecedente familiar de EA, $92.86 \%$ eran de segundo grado de consanguinidad (Tabla 4).

\section{Discusión}

La EA es una patología neurodegenerativa progresiva primaria e irreversible del sistema nervioso central, siendo la principal causa de demencia (14). La edad es el factor de riesgo mas importante para EA, específicamente por encima de los 65 años de edad, por lo que a mayor edad existirá una mayor prevalencia e incidencia de la enfermedad $(1,15)$. En

Tabla 3. Caracterización de variables genéticas en pacientes con diagnóstico de novo de DTA según edad de aparición en el Hospital Universitario C.A.R.I. sede salud mental en un periodo de dos años.

\begin{tabular}{|l|c|c|c|c|}
\hline \multirow{2}{*}{ Variable } & \multicolumn{4}{|c|}{ Tipo de aparición de EA } \\
\hline \multirow{2}{*}{ Polimorfismo APOE } & \multicolumn{2}{|c|}{ Precoz (n=7) } & \multicolumn{2}{c|}{ Tardío (n=32) } \\
\cline { 2 - 5 } & Número & $(\%)$ & Número & $(\%)$ \\
\cline { 2 - 5 }$\varepsilon 2 / \varepsilon 2$ & 0 & 0.00 & 1 & 3.13 \\
$\varepsilon 2 / \varepsilon 3$ & 1 & 14.28 & 1 & 3.13 \\
$\varepsilon 2 / \varepsilon 4$ & 1 & 14.28 & 5 & 15.62 \\
$\varepsilon 3 / \varepsilon 3$ & 1 & 14.28 & 4 & 12.50 \\
$\varepsilon 3 / \varepsilon 4$ & 4 & 57.16 & 21 & 65.62 \\
$\varepsilon 4 / \varepsilon 4$ & 0 & 0.00 & 0 & 0.00 \\
\hline
\end{tabular}

Tabla 2. Caracterización de variables de laboratorio en pacientes con diagnóstico de novo de DTA según edad de aparición en el Hospital Universitario C.A.R.I. sede salud mental en un periodo de dos años.

\begin{tabular}{|c|c|c|c|c|}
\hline \multirow[b]{4}{*}{$\begin{array}{l}\text { Glicemia en ayunas } \\
\text { Normal } \\
\text { Alteración glucosa } \\
\text { Hiperglicemia }\end{array}$} & \multicolumn{4}{|c|}{ Tipo de aparición de EA } \\
\hline & \multicolumn{2}{|c|}{$\operatorname{Precoz}(n=7)$} & \multicolumn{2}{|c|}{ Tardío (n=32) } \\
\hline & Número & $(\%)$ & Número & $(\%)$ \\
\hline & $\begin{array}{l}7 \\
0 \\
0\end{array}$ & $\begin{array}{c}100.00 \\
0.00 \\
0.00\end{array}$ & $\begin{array}{c}27 \\
3 \\
2\end{array}$ & $\begin{array}{c}84.38 \\
9.37 \\
6.25\end{array}$ \\
\hline $\begin{array}{l}\text { Nivel de colesterol total } \\
\text { Deseable } \\
\text { Límite alto } \\
\text { Alto }\end{array}$ & $\begin{array}{l}4 \\
3 \\
0\end{array}$ & $\begin{array}{c}57.14 \\
42.86 \\
0.00\end{array}$ & $\begin{array}{c}18 \\
8 \\
6\end{array}$ & $\begin{array}{l}56.25 \\
25.00 \\
18.75\end{array}$ \\
\hline $\begin{array}{l}\text { Nivel de colesterol HDL } \\
\text { Bajo } \\
\text { Intermedio } \\
\text { Alto }\end{array}$ & $\begin{array}{l}2 \\
3 \\
2\end{array}$ & $\begin{array}{l}28.57 \\
42.86 \\
28.57\end{array}$ & $\begin{array}{c}4 \\
20 \\
8\end{array}$ & $\begin{array}{l}12.50 \\
62.50 \\
25.00\end{array}$ \\
\hline $\begin{array}{l}\text { Nivel de colesterol LDL } \\
\text { Óptimo } \\
\text { Casi óptimo } \\
\text { Límite alto } \\
\text { Alto } \\
\text { Muy alto }\end{array}$ & $\begin{array}{l}1 \\
4 \\
1 \\
1 \\
0\end{array}$ & $\begin{array}{c}14.29 \\
57.13 \\
14.29 \\
14.29 \\
0.00\end{array}$ & $\begin{array}{c}6 \\
17 \\
4 \\
4 \\
1\end{array}$ & $\begin{array}{c}18.75 \\
53.12 \\
12.50 \\
12.50 \\
3.13\end{array}$ \\
\hline $\begin{array}{l}\text { Nivel de triglicéridos } \\
\text { Normal } \\
\text { Límite alto de la normalidad } \\
\text { Elevado }\end{array}$ & $\begin{array}{l}5 \\
1 \\
1\end{array}$ & $\begin{array}{l}71.42 \\
14.29 \\
14.29\end{array}$ & $\begin{array}{c}24 \\
5 \\
3\end{array}$ & $\begin{array}{c}75.00 \\
15.63 \\
9.37\end{array}$ \\
\hline
\end{tabular}


Tabla 4. Caracterización de variables de antecedentes personales, tóxicos y familiares en pacientes con diagnóstico de novo de DTA según edad de aparición en el Hospital Universitario C.A.R.I. sede salud mental en un periodo de dos años.

\begin{tabular}{|c|c|c|c|c|}
\hline \multirow[b]{4}{*}{$\begin{array}{l}\text { Antecedente de tabaquismo } \\
\mathrm{Si} \\
\text { No }\end{array}$} & \multicolumn{4}{|c|}{ Tipo de aparición de EA } \\
\hline & \multicolumn{2}{|c|}{$\operatorname{Precoz}(n=7)$} & \multicolumn{2}{|c|}{ Tardío (n=32) } \\
\hline & Número & $(\%)$ & Número & $(\%)$ \\
\hline & $\begin{array}{l}4 \\
3\end{array}$ & $\begin{array}{l}57.14 \\
42.86\end{array}$ & $\begin{array}{l}19 \\
13\end{array}$ & $\begin{array}{l}59.37 \\
40.63\end{array}$ \\
\hline $\begin{array}{l}\text { Tiempo tabaquismo de fumadores } \\
\text { Entre } 1 \text { y } 5 \text { años } \\
\text { Mayor de } 5 \text { años }\end{array}$ & $\begin{array}{l}1 \\
3\end{array}$ & $\begin{array}{l}25.00 \\
75.00\end{array}$ & $\begin{array}{c}5 \\
14\end{array}$ & $\begin{array}{l}26.32 \\
73.68\end{array}$ \\
\hline $\begin{array}{l}\text { Antecedente de hipertensión } \\
\mathrm{Si} \\
\text { No }\end{array}$ & $\begin{array}{l}3 \\
4\end{array}$ & $\begin{array}{l}42.85 \\
57.15\end{array}$ & $\begin{array}{l}15 \\
17\end{array}$ & $\begin{array}{l}46.87 \\
53.13\end{array}$ \\
\hline $\begin{array}{l}\text { Antecedente de diabetes mellitus } \\
\mathrm{Si} \\
\mathrm{No}\end{array}$ & $\begin{array}{l}0 \\
7\end{array}$ & $\begin{array}{c}0.00 \\
100.00\end{array}$ & $\begin{array}{c}4 \\
28\end{array}$ & $\begin{array}{l}12.50 \\
87.50\end{array}$ \\
\hline $\begin{array}{l}\text { Antecedente de hiperlipidemia } \\
\mathrm{Si} \\
\text { No }\end{array}$ & $\begin{array}{l}2 \\
5\end{array}$ & $\begin{array}{l}28.57 \\
71.43\end{array}$ & $\begin{array}{c}8 \\
24\end{array}$ & $\begin{array}{l}25.00 \\
75.00\end{array}$ \\
\hline $\begin{array}{l}\text { Antecedente familiar de demencia } \\
\mathrm{Si} \\
\text { No }\end{array}$ & $\begin{array}{l}5 \\
2\end{array}$ & $\begin{array}{l}71.43 \\
28.57\end{array}$ & $\begin{array}{l}19 \\
13\end{array}$ & $\begin{array}{l}59.37 \\
40.63\end{array}$ \\
\hline $\begin{array}{l}\text { Antecedente familiar de demencia primer grado } \\
\mathrm{Si} \\
\text { No }\end{array}$ & $\begin{array}{l}5 \\
0\end{array}$ & $\begin{array}{c}100.00 \\
0.00\end{array}$ & $\begin{array}{c}7 \\
12\end{array}$ & $\begin{array}{l}36.84 \\
63.16\end{array}$ \\
\hline $\begin{array}{l}\text { Antecedente familiar de demencia segundo grado } \\
\mathrm{Si} \\
\mathrm{No}\end{array}$ & $\begin{array}{l}0 \\
5\end{array}$ & $\begin{array}{c}0.00 \\
100.00\end{array}$ & $\begin{array}{c}18 \\
1\end{array}$ & $\begin{array}{c}94.74 \\
5.26\end{array}$ \\
\hline $\begin{array}{l}\text { Antecedente familiar de EA } \\
\mathrm{Si} \\
\text { No }\end{array}$ & $\begin{array}{l}4 \\
3\end{array}$ & $\begin{array}{l}57.14 \\
42.86\end{array}$ & $\begin{array}{l}14 \\
18\end{array}$ & $\begin{array}{l}43.75 \\
56.25\end{array}$ \\
\hline $\begin{array}{l}\text { Antecedente familiar de EA de primer grado } \\
\mathrm{Si} \\
\mathrm{No}\end{array}$ & $\begin{array}{l}4 \\
0\end{array}$ & $\begin{array}{c}100.00 \\
0.00\end{array}$ & $\begin{array}{c}3 \\
11\end{array}$ & $\begin{array}{l}21.43 \\
78.57\end{array}$ \\
\hline $\begin{array}{l}\text { Antecedente familiar de EA de segundo grado } \\
\mathrm{Si} \\
\text { No }\end{array}$ & $\begin{array}{l}0 \\
4\end{array}$ & $\begin{array}{c}0.00 \\
100.00\end{array}$ & $\begin{array}{c}13 \\
1\end{array}$ & $\begin{array}{c}92.86 \\
7.14\end{array}$ \\
\hline
\end{tabular}

Colombia, la prevalencia de la demencia varía según estudios, puntos de corte para la edad y territorio con cifras entre 1.3 y $39.4 \%$ (16-20). Las regiones suroccidental y oriental de Colombia tienen la mayor prevalencia de demencia en los individuos de 50 y 70 años $(4,16)$.

Dentro de las dos formas de aparición de DTA, se encuentran los casos de aparición precoz y tardía. La forma familiar o precoz se manifiesta antes de los 65 años de edad, presenta una progresión rápida, tiene un patrón de herencia autosómico dominante y está relacionado con mutaciones en el gen de la proteína precursora amiloide, Presenilina 1 o Presenilina 2. La forma esporádica o tardía se manifiesta con edad de inicio mayor a los 65 años, es influenciada por interacción entre factores de susceptibilidad y medioambientales y es de aparición gradual, con progresión lenta y predominio de deterioro de la memoria sobre discapacidad intelectual $(1,2,9,21)$. En nuestra investigación se observó que la población con DTA de inicio tardío era mayor que la población precoz, similar a lo reportado en la literatura (21).
En los resultados arrojados por el presente estudio, se evidenció que el sexo femenino fue más frecuente en la población general y según edad de aparición, similar a lo reportado en la literatura, puesto que las mujeres por encima de 55 años tienen doble riesgo de padecer demencia por mayor expectativa de vida e incidencia de demencia a edades más avanzadas $(6$, 22). Las diferencias de género pueden deberse a diferencias biológicas, de supervivencia o diferencias en el comportamiento y exposición de los individuos (23), ocurriendo el mayor efecto en portadores de APOE $\varepsilon 4(24,25)$.

En nuestra investigación, en el grupo de DTA precoz, la mayoría de los pacientes contaban con compañía habitual en su hogar, mientras que los pacientes con DTA tardío eran viudos más frecuentemente, que hace pensar que el ámbito social puede jugar un rol en el diagnóstico temprano y abordaje a tiempo de la enfermedad. Los individuos con mayor número de relaciones sociales tienen menor incidencia de demencia y una detección temprana de sintomatología, debido a que un familiar puede notar cambios en la función cognitiva, comportamiento y funcionamiento del individuo 
(26). En pacientes con DTA precoz y casados, la presencia de un compañero lleva a un diagnóstico en fases iniciales y tempranas de la enfermedad (27).

En el presente estudio, se evidenció que el sobrepeso fue más frecuente en la población con DTA precoz que tardío. El sobrepeso se ha asociado con disminución de la edad de aparición de EA(28). La adiposidad produce deterioro cognitivo y degradación neuronal, primordialmente en personas con gran nivel de inflamación (29). Así mismo la obesidad afecta funciones cognitivas y motoras $(30,31)$, debido a la alteración del metabolismo de la insulina, cuyos receptores se expresan densamente en la región temporal, frontal e hipocampo, que se encargan de la formación de memoria $(32,33)$. Los niveles de glicemia en el presente estudio se encontraron dentro del rango de normalidad para la totalidad del grupo precoz y la mayoría del grupo tardío, a pesar de que la hiperglicemia produce inflamación tisular y aumento del estrés oxidativo y productos glicosilados (34) en pacientes con EA comparados con individuos sanos (32).

Dentro de las medidas de laboratorio, la presencia del nivel de colesterol LDL por fuera del rango óptimo y del colesterol HDL fuera del rango alto, fue frecuente tanto en grupo precoz como tardío. Se ha reportado la asociación entre DTA precoz y niveles elevados de colesterol LDL, incluso independiente de APOE (35). Niveles altos de HDL se asocian con menor riesgo de demencia y tanto EA probable y EA posible (36), dado que su efecto protector se basa en la reducción de neuroinflamación y protección en contra del déficit de memoria (37). En el presente estudio, los niveles de triglicéridos se encontraron mas frecuentemente en el rango de normalidad para ambos grupos de DTA, a pesar de que niveles séricos de triglicéridos se han encontrado elevados en pacientes con EA, sugiriendo un factor de riesgo potencial para alteración cognitiva (38). El gen de la apolipoproteína $\mathrm{E}(A P O E)$, es el único gen que confiere susceptibilidad al individuo para las formas, tanto esporádicas como familiares de DTA $(2,21)$. El gen APOE, localizado en el brazo largo del cromosoma 19 (19q13.2) presenta tres alelos $(\varepsilon 2, \varepsilon 3, y \varepsilon 4)$, que se transmiten de forma codominante y codifican para tres isoformas de proteínas (E2, E3 y E4), que modifican la estructura proteica y la afinidad al receptor, afectando el catabolismo lipídico (39, 40). Se diferencian por cambio de nucleótido C/T en el exón 4 en las posiciones 112 y 158 de la secuencia aminoacídica (cambio de arginina o cisteína) y resultan en seis genotipos posibles: $\varepsilon 2 / \varepsilon 2, \varepsilon 3 / \varepsilon 3, \varepsilon 2 / \varepsilon 3, \varepsilon 3 / \varepsilon 4, \varepsilon 2 / \varepsilon 4$ y $\varepsilon 4 / \varepsilon 4$ (41).

El genotipo más frecuentemente reportado en la población general de Barranquilla, Medellín, Quindío, Bogotá y Valle del Cauca (Colombia) es el $\varepsilon 3 / \varepsilon 3$, seguido por $\varepsilon 3 / \varepsilon 4$, $\varepsilon 2 / \varepsilon 3$ y $\varepsilon 2 / \varepsilon 4$ generalmente (42). La presencia de los genotipos $\varepsilon 4 / \varepsilon 4$ y $\varepsilon 2 / \varepsilon 2$ en la población general varía según la ciudad colombiana (42). En el presente estudio, tanto en la población con DTA como en los grupos precoz y tardío, ser heterocigoto para el alelo $\varepsilon 4$ fue el hallazgo más frecuente. En la población con DTA, el genotipo más frecuente fue $\varepsilon 3 /$ $\varepsilon 4$, seguido por $\varepsilon 2 / \varepsilon 4, \varepsilon 3 / \varepsilon 3, \varepsilon 2 / \varepsilon 3$ y finalmente $\varepsilon 2 / \varepsilon 2$. En el grupo con DTA precoz el genotipo más frecuente fue $\varepsilon 3 / \varepsilon 4$, seguido equitativamente por $\varepsilon 2 / \varepsilon 3, \varepsilon 2 / \varepsilon 4$ y $\varepsilon 3 / \varepsilon 3$, mientras que en el grupo con DTA tardío el genotipo más frecuente fue $\varepsilon 3 / \varepsilon 4$, seguido por $\varepsilon 2 / \varepsilon 4, \varepsilon 3 / \varepsilon 3$ y finalmente equitativo entre $\varepsilon 2 / \varepsilon 2$ y $\varepsilon 2 / \varepsilon 3$.

A pesar de que no se evidenciaron homocigotos para $\varepsilon 4$ en ambos grupos, se observaron pacientes homocigotos para $\varepsilon 3$, que se considera el alelo más común en la población general. El alelo $\varepsilon 4$ se asocia a bajo rendimiento neurocognitivo, particularmente en memoria episódica y aumenta el riesgo de desarrollar DTA tardío (43). El alelo $\varepsilon 4$ se considera una variante de riesgo comparado con el alelo $\varepsilon 3$ (neutral) y $\varepsilon 2$ (protector), por lo que se considera que $A P O E$ $\varepsilon 4$ es el mayor factor de riesgo genético para el desarrollo de EA (44-46). Ser portador de un alelo $\varepsilon 4$ incrementa el riesgo para EA de tres a diez veces (1), disminuye la edad de aparición para el grupo de DTA tardío (47) por una o dos décadas (48) y puede influir en el incremento de niveles de colesterol total, LDL y triglicéridos (49). Se evidenció en el estudio un caso homocigoto de $\varepsilon 2$ en la población con DTA tardío, el genotipo más raro de la población general. Los portadores de $\varepsilon 2$ tienen menor riesgo y un retraso en la edad de inicio de EA, comparado con homocigotos para $\varepsilon 3$ y portadores de $\varepsilon 4$ (41).

Dentro de los antecedentes personales de nuestro estudio, el hábito de tabaquismo específicamente por más de cinco años, fue observado por la mayoría de los pacientes tanto precoces como tardíos. El tabaquismo duplica el riesgo de desarrollar demencia y EA, debido a que acelera el deterioro de funciones cognitivas, induce cambios sinápticos y genera neurodegeneración en fases tempranas (50). El tabaquismo se asocia a cortezas más delgadas en regiones que demuestran atrofia significativa en DTA precoz (51). En el estudio fue más frecuente no tener antecedente de hipertensión arterial para ambos grupos. La presión arterial elevada precede la EA por décadas, pero disminuye en los años anteriores al inicio de la demencia (52).

En el presente estudio, para ambos grupos fue más frecuente el antecedente familiar de demencia, siendo de primer grado de consanguinidad para el grupo precoz y de segundo grado para el grupo tardío. En el grupo precoz fue más frecuente el antecedente familiar de EA, siendo en su totalidad de primer grado de consanguinidad. La heredabilidad para la EA es elevada (53), debido a que familiares de primer grado (padres e hijos) de un individuo con EA tienen un riesgo de $20 \%$ de desarrollar la enfermedad (54). Se ha asociado mayor alteración de memoria y adelgazamiento cortical con historia familiar de primer grado de EA (55). La historia familiar de demencia se asocia con disminución de materia gris en regiones afectadas típicamente por EA (56). En el grupo tardío de nuestro estudio, el antecedente familiar de EA fue más frecuente con familiares de segundo grado de consanguinidad. Cuando varios individuos en una familia tienen EA, el riesgo se incrementa (54). 
Dentro de las limitaciones del estudio, existió sesgo de selección dado que fue un muestreo de conveniencia de tipo secuencial, debido a que los datos obtenidos son de un hospital departamental que limita la generalización de los resultados en la población de la región Caribe. Por el tamaño de muestra y la metodología descriptiva del estudio, no se confirman asociaciones entre los factores estudiados y el deterioro cognitivo, pero se caracteriza, aporta y compara el conocimiento con lo que otros estudios estadísticos han evidenciado. La escolaridad, tiempo de vigencia laboral y sedentarismo no fueron incluidas como variables en el estudio. Se generó variabilidad en cuanto a los laboratorios, debido a que fueron realizados según la afiliación del paciente a su entidad promotora de salud.

\section{Conclusiones}

Por medio del presente estudio, se lograron identificar y caracterizar factores de riesgo en pacientes con DTA en un hospital departamental en Barranquilla, Colombia. La edad es el principal factor asociado a la aparición de DTA, por lo cual fue más frecuente el hallazgo de DTA tardío que precoz en la población estudiada. El sexo femenino fue más frecuente en ambos grupos al igual que lo reportado. Las relaciones sociales juegan un rol importante en la identificación temprana de la sintomatología, según lo observado en la presente investigación. El sobrepeso fue mas frecuente en la población con DTA precoz y se ha asociado con disminución de la edad de aparición de EA. Las alteraciones de los lípidos evidenciados en ambos grupos de nuestro estudio ya han sido reportados y asociados con riesgo de demencia y EA. Lo evidenciado por el estudio genético soporta la teoría de que tener al menos un alelo $\varepsilon 4$ del gen $A P O E$ es un hallazgo frecuente en la DTA, mientras que el genotipo más frecuente de la población general es ser homocigoto para el alelo $\varepsilon 3$. El tabaquismo, específicamente por más de cinco años, fue evidenciado en ambos grupos, el cual se ha reportado como riesgo para el desarrollo de demencia y EA. Finalmente, en el grupo precoz se observó más frecuentemente el antecedente familiar de demencia y Alzheimer en primer grado de consanguinidad, comparado con lo observado en el grupo tardío. Por lo anterior, el estudio del polimorfismo del gen $A P O E$ junto con el conocimiento de variables demográficas, socioculturales, de laboratorio y antecedentes, son una herramienta útil para evaluar la presencia de características relacionadas con la DTA, según la edad de aparición. Se necesitan más estudios en el Caribe colombiano que evalúen la asociación entre los factores de riesgo y la DTA según la edad de aparición. Con el presente estudio se pueden hacer comparaciones con otras investigaciones que permitan a los profesionales de salud lograr un abordaje adecuado y temprano.

\section{Referencias}

1. Lopera F.Enfermedad de Alzheimer Familiar. Revista Neuropsicología, Neuropsiquiatría y Neurociencias. 2012;12(1):163-88.
2. Ardila A, Rosselli M. Capítulo 13: Envejecimiento Normal y Patológico: Demencia de tipo Alzheimer. Neuropsicología Clínica. 2007;240-7.

3. Alzheimer's Disease International. Informe Mundial sobre el Alzheimer 2019 Actitudes hacia la demencia. Londres: Alzheimer's Disease International [Internet]. 2019. [citado el 11 de diciembre de 2019]; 1-13. Disponible en: https://www.alz. co.uk/research/WorldAlzheimerReport2019-Spanish-Summary.pdf

4. Ruiz C, Nariño D, Muñoz Joe. Epidemiología y carga de la Enfermedad de Alzheimer. Acta Neurol Colomb. 2010;26(3):87-94.

5. Llibre JJ, Guerra MA. Enfermedad de Alzheimer. Situación actual y estrategias terapéuticas. Rev Cubana Med. 1999;38(2):134-42.

6. Prieto JC, Ortiz M, López de Silanes M, Llanero M. Impacto social de la enfermedad de Alzheimer y otras demencias. Fundación Española de Enfermedades Neurológicas [Internet]. 2011 [citado el 6 de junio de 2018]:1-47. Disponible en:http://www.fundaciondelcerebro.es/docs/imp_social_alzheimer.pdf

7. alcour VG, Masaki KH, Curb JD, Blanchette PL. The detection of dementia in the primary care setting. Arch Intern Med. 2000;160(19):2964-8.

8. McKhann GM, Knopman DS, Chertkow H, Hyman BT, Jack CR, Kawas CH et al. The diagnosis of dementia due to Alzheimer's disease: Recommendations from the National Institute on Aging-Alzheimer's Association workgroups on diagnostic guidelines for Alzheimer's disease. Alzheimers Dement. 2011;7(3):2639.

9. Clinical descriptions and diagnostic guidelines. The ICD-10 Classification of Mental and Behavioural Disorders. World Health Organization [Internet]. 1993 [citado el 6 de junio de 2018];42-9. Disponible en: www.who.int/classifications/ icd/en/GRNBOOK.pdf

10. Dubois B, Hampel H, Feldmann HH, Scheltens P, Asien P, Andrieu S et al. Preclinical Alzheimer's disease: Definition, natural history, and diagnostic criteria. Alzheimers Dement. 2016;12(3):292-323.

11. Rubio MA, Moreno C, Cabrerizo L. Guías para el tratamiento de las dyslipidemias en el adulto: Adult Treatment Panel III (ATP-III). Endocrinol Nutr. 2004;51(5):254-65.

12. American Diabetes Association. Standards of Medical Care in Diabetes-2019. The Journal of Clinical and Applied Research and Education [Internet]. 2019 [citado el 11 de diciembre de 2019];42(1): S13-28. Disponible en: https://care. diabetesjournals.org/content/diacare/suppl/2018/12/17/42.Supplement_1.DC1/ DC_42_S1_2019_UPDATED.pdf

13. Hixson JM, Vernier DT. Restriction isotyping of human apolipoprotein E by gene amplification and cleavage with Hha I. J Lipid Res. 1990;31(3):545-8.

14. Donoso, A. La enfermedad de Alzheimer. Rev chil neuro-psiquiatr. 2003;41(2):1322.

15. Querfurth H, LaFerla FM. Alzheimer's disease. New Engl J Med. 2010;362(4):329-44.

16. Pradilla G, Vesga BE, León-Sarmiento FE, Grupo GENECO. Estudio neuroepidemiológico nacional (EPINEURO) colombiano. Rev Panam de Salud Publ. 2003;14(2):104-11.

17. Díaz R, Marulanda F, Martínez MH. Prevalencia de deterioro cognitivo y demencia en mayores de 65 años en una población urbana colombiana. Acta Neurol Colomb. 2013;29(3):141-51.

18. Pradilla G, Vesga BE, Díaz LA, Pinto NX, Sanabria CL, Baldovino BP, et al Estudio neuroepidemiológico en la comunidad urbana de Piedecuesta Santander. Acta Med Colomb. 2002; 27:407-20.

19. Díaz-Cabezas R, Ruano-Restrepo MI, Chacón-Cardona JA, Vera-González A. Perfil neuroepidemiológico en la zona centro del departamento de Caldas (Colombia), años 2004- 2005. Rev Neurol. 2006;43:646-52.

20. Gooding MP, Amaya E, Parra M, Ríos AM. Prevalencia de las demencias en el municipio de Neiva 2003-2005. Acta Neurol Colomb. 2006;22:243-8.

21. Setó-Salvia N, Clarimón J. Genética en la enfermedad de Alzheimer. Rev Neurol. 2010;50(6):360-4

22. Ott A, Breteler MM, van Harskamp F, Stijnen T, Hofman A. Incidence and risk of dementia. The Rotterdam study. Am J Epidemiol. 1998;147(6):574-80.

23. Launer LJ, Andersen K, Dewey ME, Lettenneur L, Ott A, Amaducci LA et al. Rates and risk factors for dementia and Alzheimer's disease. Results from EURODEM pooled analyses. EURODEM Incidence Research Group and Work Groups. European Studies of Dementia. Neurology. 1999;52(1):78-84.

24. Lin KA, Choudhury KR, Rathakrishnan BG, Marks DM, Petrella JR, Doraiswamy $P M$ et al. Marked gender differences in progression of mild cognitive impairment over 8 years. Alzheimers Dement. 2015;1(2):103-10.

25. Xing Y, Tang Y, Jia J. Sex Differences in Neuropsychiatric Symptoms of Alzheimer's Disease: The Modifying Effect of Apolipoprotein E $\varepsilon 4$ Status. Behav Neurol. 2015;2015:1-6.

26. Dubois B, Padovani A, Scheltens P, Rossi A, Dell'Agnello G. Timely Diagnosis 
For Alzheimer's Disease: A Literature Review on Benefits and Challenges. $J$ Alzheimers Dis. 2016;49(3):617-31.

27. de Oliveira FF, Bertolucci PH, Chen ES, Smith MC. Assessment of risk factors for earlier onset of sporadic Alzheimer's disease dementia. Neurol India. 2014;62(6):625-30

28. Naderali EK, Ratcliffe SH, Dale MC. Obesity and Alzheimer's disease: A link between body weight and cognitive function in old age. Am J Alzheimers Dis Other Demen. 2009;24(6): 445-9.

29. Bermejo P, Martín-Aragón S. Enfermedad de Alzheimer. La nutrición como factor protector. Farmacia Profesional. 2008;22(5): 54-58.

30. Lafortuna CL, Agosti F, Proietti M, Adormi F, Sartorio A. The combined effect of adiposity, fat distribution and age on cardiovascular risk factors and motor disability in a cohort of obese women (aged 18-83). J Endocrinol Invest. 2006;29(10):905-12.

31. Elias MF, Elias PK, Sullivan LM, Wolf PA, D'Agostino RB. Lower cognitive function in the presence of obesity and hypertension: the Framingham heart study. Int J Obes Relat Metab Disord. 2003;27(2):260-8.

32. Watson GS, Craft S. The role of insulin resistance in the pathogenesis of Alzheimer's disease: implications for treatment. CNS Drugs. 2003;17(1):27-45.

33. Watson GS, Craft S. Modulation of memory by insulin and glucose: neuropsychological observations in Alzheimer's disease. Eur J Pharmacol. 2004;490(13):97-113

34. Botero LE, Toro AE, Patiño AJ, Salazar G, Rodríguez JC, Suárez-Escudero JC et al. Diabetes mellitus en pacientes con enfermedad de Alzheimer: descripción clínica y correlación con el genotipo $A P O E$ en una muestra de población del departamento de Antioquia, Colombia. Biomédica. 2012;32:239-51.

35. Wingo TS, Cutler DJ, Wingo AP, Le NA, Rabinovici GD, Miller BL et al. Association of Early-Onset Alzheimer Disease With Elevated Low-Density Lipoprotein Cholesterol Levels and Rare Genetic Coding Variants of APOB. JAMA Neurol. 2019;76(7):809-17.

36. Reitz C, Tang MX, Schupf N, Manly JJ, Mayeux R, Luchsinger JA. Association of Higher Levels of High-Density Lipoprotein Cholesterol in Elderly Individuals and Lower Risk of Late-Onset Alzheimer Disease. Arch Neurol. 2010;67(12): 1491-7.

37. Button EB, Robert J, Caffrey TM, Fan J, Zhao W, Wellington CL. HDL from man Alzheimer's disease perspective. Curr Opio Lipidol. 2019;30(3):224-34.

38. Liu Y, Zhong X, Shen J, Jiao L, Tong J, Zhao W et al. Elevated serum TC and LDL-C levels in Alzheimer's disease and mild cognitive impairment: A metaanalysis study. Brain Res. 2020;1727:146554.

39. Garcia AM. La apolipoproteína E: el polimorfismo genético y su relación con los cambios metabólicos, los hábitos alimenticios y el origen étnico. Rev Colomb Cardiol. 2003;10:189-93.

40. Mahley RW, Innerarity TL, Rall SC Jr, Weisgraber KH. Plasma lipoproteins: Apolipoprotein structure and function. J Lipid Res. 1984;25(12):1277-94.

41. Suri S, Heise V, Trachtenberg AJ, Mackay CE. The forgotten APOE allele:
A review of the evidence and suggested mechanisms for the protective effect of APOE e2. Neurosci Biobehav Rev. 2013;37(10):2878-86.

42. Ruiz M, Arias I, Rolón G, Hernández E, Garavito P, Silvera-Redondo C. Análisis del polimorfismo del gen APOE en la población de Barranquilla, Colombia. Biomédica. 2016;36:52-8.

43. Yasen AL, Raber J, Miller JK, Piper BJ. Sex, but not Apolipoprotein E Polymorphism, Differences in Spatial Performance in Young Adults. Arch Sex Behav. 2015;44(8):2219-26

44. Liu M, Bian C, Zhang J, Wen F. Apolipoprotein E gene polymorphism and Alzheimer's disease in Chinese population: A meta-analysis. Sci Rep. 2014;4:1-7.

45. Puglielli L, Tanzi RE, Kovacs DM. Alzheimer's disease: the cholesterol connection. Nat Neurosci. 2003;6(4):345-51

46. Reiman EM, Arboleda-Velasquez JF, Quiroz YT, Huentelman MJ, Beach TG, Caselli RJ et al. Exceptionally low likelihood of Alzheimer's Dementia in APOE2 homozygotes. Alzheimers Disease Genetics Consortium. [Internet]. 2019. [citado el 11 de Diciembre de 2019]:1-26. Disponible en: https://www.medrxiv. org/content/10.1101/19011015v1

47. Reitz C, Mayeux R. Use of genetic variation as biomarkers for mild cognitive impairment and progression of mild cognitive impairment to dementia. J Alzheimers Dis. 2010;19(1):229-51.

48. Verghese PB, Castellano JM, Holtzman DM. Apolipoprotein E in Alzheimer's disease and other neurological disorders. Lancet Neurol. 2011;10(3):241-52.

49. Torres AL, Guerra M, Segrera A, Wagner J, Alvarado M. Modulación de los niveles de lípidos y lipoproteínas por el polimorfismo de la apolipoproteína E en individuos sanos de Bogotá D.C. NOVA. 2005;3(3):31-6.

50. Ho YS, Yang, X, Yeung SC, Chiu K, Lau, CF, Tsang AW et al. Cigarette smoking accelerated brain aging and induced pre-Alzheimer-Like neuropathology in rats. PLoS ONE. 2012;7(5):e36752.

51. Durazzo TC, Meyerhoff DJ, Yoder KK. Cigarette smoking is associated with cortical thinning in anterior frontal regions, insult and regions showing atrophy in early Alzheimer's Disease. Drug Alcohol Depend. 2018;192:277-84.

52. Skoog I, Gustafson D. Hypertension, hypertension-clustering factors and Alzheimer's disease. Neurol Res. 2003;25(6):675-80.

53. Gatz M, Reynolds CA, Fratiglioni L, Johansson B, Mortimer JA, Berg S et al. Role of genes and environments for explaining Alzheimer disease. Arch Gen Psychiatry. 2006;63(2):168-74

54. Family History of Alzheimer Disease. Department of Human Genetics. Emory University School of Medicine[Internet]. 2008. [citado el 6 de junio de 2018]; 1. Disponible en: https://genetics.emory.edu/documents/resources/Emory_Human_Genetics_Family_History_Alzheimer_Disease.PDF

55. Haussmann R, Ganske S, Gruschwitz A, Werner A, Osterrath A, Lange J et al. Family History of Alzheimer's Disease and Subjective Memory Performance. Am J Alzheimers Dis Other Demen. 2018;33(7):458-62.

56. Kate M, Sanz-Arigita EJ, Tijms BM, Wink AM, Clerigue M, Garcia-Sebastian M et al. Impact of APOE- $\varepsilon 4$ and family history of dementia on gray matter atrophy in cognitively healthy middle-aged adults. Neurobiol Aging. 2016;38:14-20. 